\title{
FERMILAB BOOSTER MODELING AND SPACE CHARGE STUDY*
}

\author{
W. Chou", A. Drozhdin, P. Lucas, F. Ostiguy, FNAL, Batavia, IL 60510, USA
}

\section{Abstract}

The Fermilab Booster is a bottleneck limiting the proton beam intensity in the accelerator complex. A study group has been formed in order to have a better understanding of this old machine and seek possible improvements [1]. The work includes lattice modeling, numerical simulations, bench measurements and beam studies. Based on newly obtained information, it has been found that the machine acceptance is severely compromised by the orbit bump and dogleg magnets. This, accompanied by emittance dilution from space charge at injection, is a major cause of the large beam loss at the early stage of the cycle. Measures to tackle this problem are being pursued.

\section{INTRODUCTION}

The Fermilab Booster is a 30 years old machine and the only machine at Fermilab that has never been upgraded. It is the bottleneck in the accelerator complex limiting the proton beam intensity. The linac upstream from the Booster can deliver 5 times more protons than it does now. The Main Injector downstream from the Booster can also accept 5 times more protons. However, the Booster, which sits in between, can provide no more than $6 \mathrm{e} 12$ protons per cycle. Otherwise the loss would be prohibitive (Fig. 1). Most of the losses occur at the early stage of the cycle (about 25-30\%); in particular during the first few $\mathrm{ms}$. (The cycle time is $66.7 \mathrm{~ms}$.) In order to understand the cause of the early loss, a study group was formed about 6 months ago. It launched a systematic investigation on the Booster. A comprehensive lattice model using MAD is established. The space charge codes ESME (authored by J. MacLachlan) and ORBIT (authored by J. Holmes) are employed. With the help of the Proton Source Department and other departments/divisions, a series of beam studies and magnet field measurements are also carried out.

\section{PERTURBATION ON LINEAR OPTICS: THE DOGLEG EFFECT}

One surprise in this study (first discovered by A. Drozhdin) is that the linear optics of the Booster is significantly perturbed by the edge focusing of the injection and extraction orbit bumps. The latter is termed "dogleg" at Fermilab. As can be seen in Figure 2, in the horizontal plane, the maximum beta function is increased from $33 \mathrm{~m}$ to $47 \mathrm{~m}$, maximum dispersion from $3 \mathrm{~m}$ to 6 $\mathrm{m}$; in the vertical plane, the maximum beta from $20 \mathrm{~m}$ to $26 \mathrm{~m}$. The edge focusing strength of a bending magnet is:

$$
1 / f=\tan \theta / \rho \cong \theta^{2} / L
$$

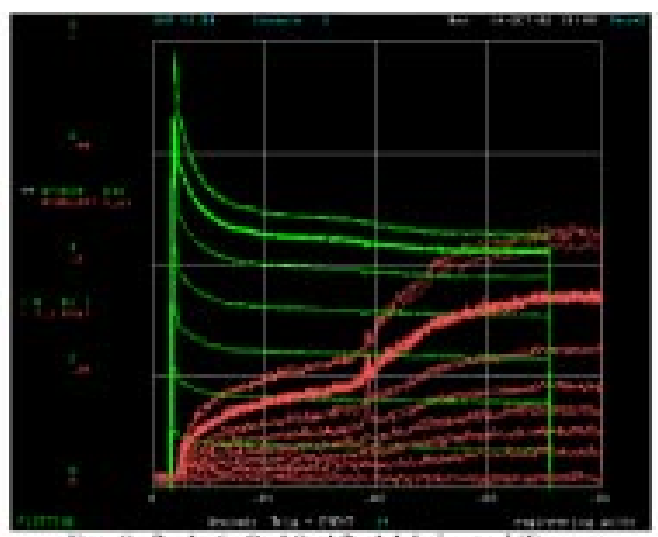

For $0,2,4,6,8,10,12,14$ Injected 1 urns

Figure 1: Proton intensity (in green) and integrated beam power loss (in red) during the cycle. (courtesy R. Webber)
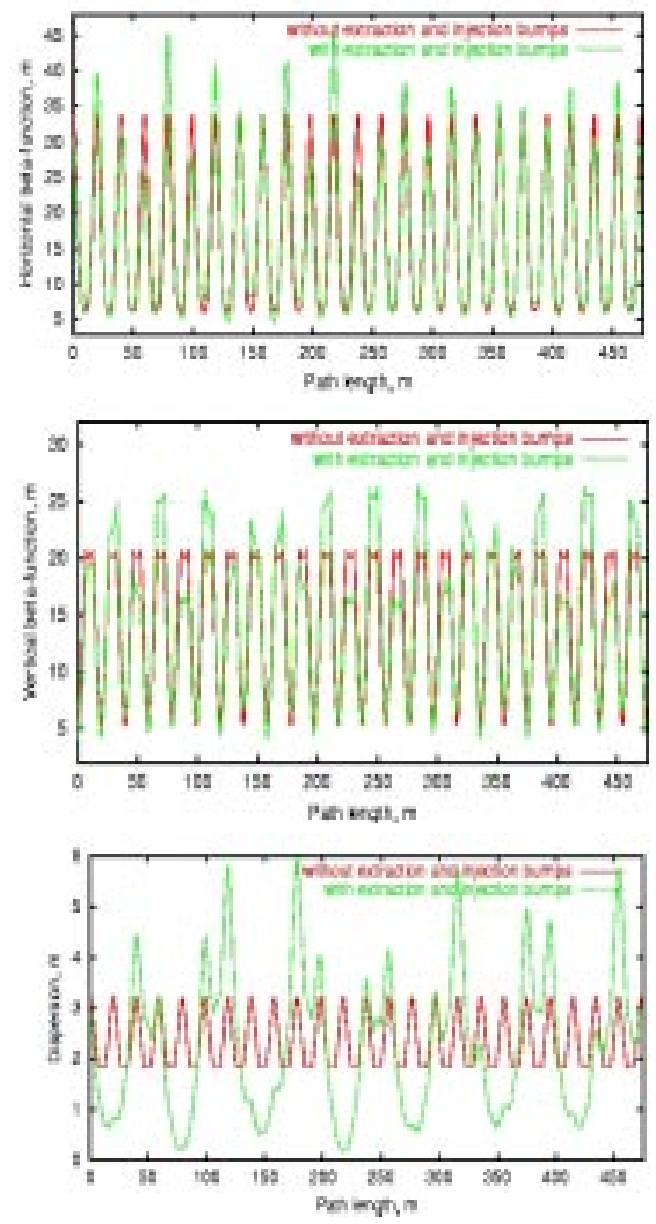

Figure 2: Lattice function perturbation due to edge focusing of the orbit bump and dogleg. Red: original, green: perturbed. Top $-\beta_{x}$, middle $-\beta_{y}$, bottom $-D_{x}$. 
in which $\theta$ is the bending angle, $\rho$ the bending radius, $L$ the magnet length. For the Booster doglegs, $L$ is small $(0.2 \mathrm{~m})$ and $\theta$ large $(60 \mathrm{mrad})$. There are two doglegs, each with 4 bending magnets. The focusing effects are additive, giving rise to a significant amount of extra focusing $\left(0.1152 \mathrm{~m}^{-1}\right.$, close to one main magnet which has $\left.1 / f=0.1567 \mathrm{~m}^{-1}\right)$ and leading to a big perturbation to the linear lattice. Both the injection orbit bump (horizontal bend) and extraction doglegs (vertical bend) are rectangular bends. Therefore, their edge focusing acts in the non-deflecting plane. That is, vertical for the injection orbit bump and horizontal for the dogleg. The former is pulsed (pulse length about $150 \mu \mathrm{s}$ ), while the latter DC. Hence, the doglegs cause more damage to the beam.

This effect was quickly confirmed in a beam study. The measured tune shift and dispersion perturbation are in good agreement with the MAD prediction. When one of the doglegs was removed in a machine experiment, the beam transfer efficiency showed a considerable improvement (Fig. 3). A milestone of the MiniBooNE neutrino program (5e16 protons per hour) was reached. There was a champagne celebration.

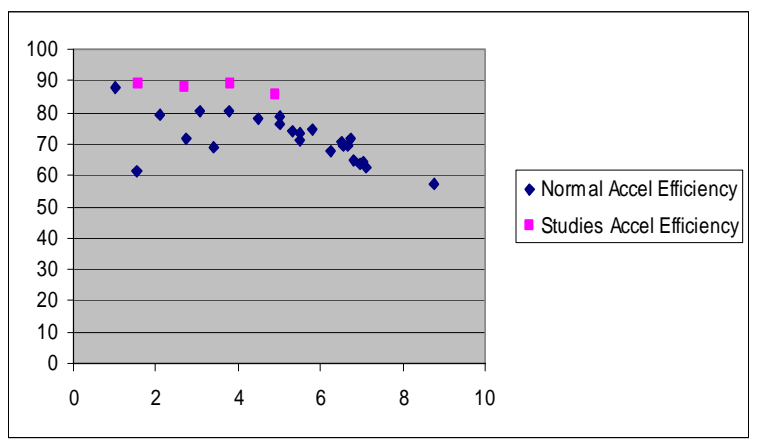

Figure 3: The beam transfer efficiency is improved when one of the doglegs is removed during a machine study. The $\mathrm{x}$-axis is the injected number of protons $\left(\times 10^{12}\right)$. Blue - normal operation; pink - one dogleg removed. (courtesy J. Lackey)

\section{CHROMATICITY MODELING}

The present setting of the chromaticity during the cycle is somewhat confusing. For instance, the horizontal chromaticity is positive below transition, which could cause the head-tail instability that has not been seen. It is decided to try other chromaticity ramp curves by compiling a spreadsheet relating the sextupole setting with the machine chromaticity. There are four major contributors to the chromaticity: lattice (main quads), dogleg (edge focusing), sextupole of the main magnets and chromaticity sextupoles.

$\xi=\xi($ lat $)+\xi($ dogleg $)+\xi($ mag sext $)+\xi($ chrom sext $)$

The direct contribution form the dogleg is small. However, it has big impact on the chromaticity, because it changes the local beta and dispersion functions at the chromaticity sextupoles. One unknown parameter in this equation is the sextupole component of the main magnets, which comes not only from the body but also from the ends. In order to get a reliable value of this parameter, a "blind check" method was applied. Two teams, one working on the chromaticity and another on the field, carried out the measurements independently without communication between them. The results were then put on the table for a comparison. The agreement is very good, as listed in Table 1. It is seen that the ends compensate the body sextupole of the F magnet almost perfectly, but nearly doubles that of the D magnet.

Table 1: Sextupole component of the main magnets

\begin{tabular}{|c|c|c|c|}
\hline $\begin{array}{c}\text { Magnet } \\
\text { type }\end{array}$ & $\begin{array}{c}\text { Body } \\
\text { only }\end{array}$ & $\begin{array}{c}\text { Body + Ends } \\
\text { field meas. }\end{array}$ & $\begin{array}{c}\text { Body + Ends } \\
\text { chrom meas. }\end{array}$ \\
\hline F & 0.026 & 0.0045 & -0.003 \\
\hline D & -0.021 & -0.0413 & -0.0454 \\
\hline
\end{tabular}

\section{SPACE CHARGE STUDY}

Space charge is a dominant factor limiting the beam intensity in low energy proton machines. It causes tune shift, emittance growth and resonance. Analytical tools have limited use for this complicated phenomenon. Therefore, we invoke numerical simulations. Two codes, ESME (longitudinal) and ORBIT (transverse) are employed for this purpose. (Another group also uses a code called Synergia.)

Figure 4 shows $805 \mathrm{MHz}$ micro-bunch injected into the Booster simulated by ESME. The energy spread matches the measured value $( \pm 0.88 \mathrm{MeV})$. Figure 5 is the tune footprint obtained from ORBIT. The tune spread $(-0.3)$ agrees with that predicted by the Laslett formula. Figure 6(a) is the transverse emittance growth simulated by ORBIT. It shows two distinct regions. One is a fast growth during the 10-turn injection, another a slow growth after injection. Measurement from the ion profile monitor (IPM) seems to support this observation, see Figure 6(b). (Note: The IPM data processing is complicated and sometimes even controversial. Fig. 6(b) shows the raw data. But the processed data demonstrates a similar qualitative behavior, namely, a fast growth during injection and a slowdown after that [2].)

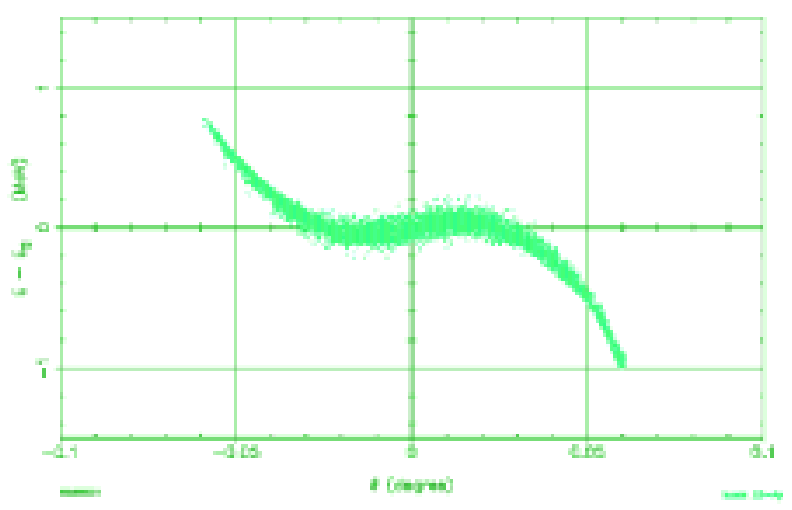

Figure 4: $805 \mathrm{MHz}$ micro-bunch simulated by ESME. 


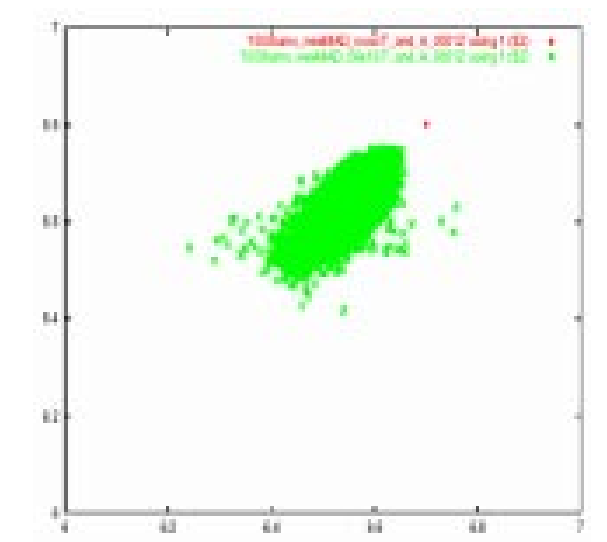

Figure 5: Tune footprint due to space charge simulated by ORBIT. The red point is the nominal tune $(6.7,6.8)$.
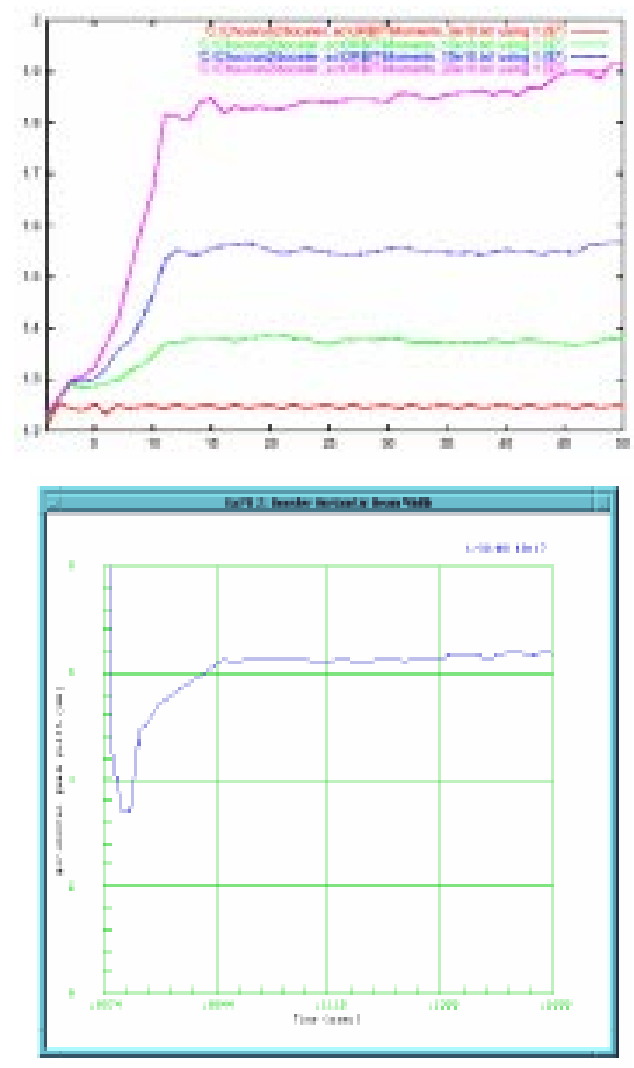

Figure 6: (a) Top: Emittance dilution during the first 50 turns from ESME. The injection time is 10 turns. From the bottom trace to the top, the space charge effect (beam intensity) is increased. (b) Bottom: Emittance dilution during the first 50 turns from IPM measurement. The first injection starts at the valley of the curve. The injection time is 10 turns.

\section{BEAM LOSS IN THE FIRST FEW MS}

Based on the results in the previous sections, one can develop a coherent picture of what happens in the first few $\mathrm{ms}$ in the Booster and why the large beam loss should occur.

\section{Longitudinal Loss}

The measured Booster longitudinal acceptance is small $( \pm 0.15-0.2 \%)$. It is about the same as the linac beam momentum spread $( \pm 0.13 \%)$. When the RF is turned on to capture the beam adiabatically, the momentum spread of the bunched beam will increase to about $\pm 0.3 \%$ and exceed the acceptance, resulting in loss.

\section{Transverse Loss}

The machine transverse acceptance is:

$$
\mathrm{A}=\left\{\beta_{\max } \times \varepsilon_{\mathrm{N}} / \beta \gamma\right\}^{-1 / 2}+\mathrm{D}_{\max } \times \Delta \mathrm{p} / \mathrm{p}+\text { c.o.d. }
$$

in which c.o.d. is the closed orbit distortion. Take the horizontal plane as an example. The magnet good field region is small $(\sim \pm 1.2$ inches $)$. For the regular values of $\beta_{\max }$ and $D_{\max }$, the maximum allowable $\varepsilon_{\mathrm{N}}$ is about $16 \pi$ mm-mrad. However, the dogleg effect blows up the lattice functions and reduces the acceptable $\varepsilon_{\mathrm{N}}$ to $8 \pi$, whereas the incoming linac beam is about $7 \pi$. The situation is worsened by the space charge. It dilutes the emittance during multi-turn injection. The beam is scraped transversely, resulting in loss.

These losses are most severe in the first few ms. When beam energy goes up, the situation improves rapidly due to a number of factors: the relative momentum spread $\Delta \mathrm{p} / \mathrm{p}$ becomes smaller, the dogleg effect $\left(\propto 1 / \gamma^{2}\right)$ and space charge effect $\left(\propto 1 / \beta \gamma^{2}\right)$ reduce quickly, the beam size also shrinks from adiabatic damping.

A systematic investigation of various measures for correcting the dogleg effect, reducing the space charge and increasing the machine acceptance is under way. This will be the content of another paper that will be published later.

\section{ACKNOLEDGEMENT}

This study is a collaboration involving physicists and engineers from the Beam Physics Department, Proton Source Department, Operation Department, Technical Division and Computer Division. The authors would like to express their thanks to the following people for their help and stimulating discussions: J. Amundson, C. Ankenbrandt, J. DiMarco, M. Foley, D. Harding, J. Lackey, J. MacLachlan, E. Malamud, E. McCrory, M. Popovic, E. Prebys, P. Schlabach, P. Spentzouris, M. Syphers, L. Teng, R. Tomlin, D. Wolff and X. Yang.

\section{REFERENCES}

[1] http://www-bd.fnal.gov/pdriver/booster/

[2] P. Spentzouris, http://www-bd.fnal.gov/pdriver/ booster/meetings2/spentz_24apr03.pdf

\footnotetext{
*Work supported by the Universities Research Association, Inc. under contract No. DE-AC02-76CH03000 with the U.S. Dept. of Energy. \#chou@fnal.gov
} 